\title{
Design of a Media Resource Management System for Colleges Based on Cloud Service
}

\author{
https://doi.org/10.3991/ijet.v15i21.18195 \\ Heng Sun, Wan Ni ( ${ }^{\bowtie}$, Peng Zhao \\ Shandong University, Jinan, China \\ niwanesdu.edu.cn
}

\begin{abstract}
In recent years, many new technologies, such as the Internet, cloud computing, and the 5th-generation $(5 \mathrm{G})$ mobile network, have been widely applied in colleges. The structure and functions of traditional media technology can no longer satisfy the demand for media resource management (MRM) in colleges. Therefore, this paper creates an intelligent MRM system based on cloud service, providing a brand-new intelligent MRM solution. Through cloud service and artificial intelligence (AI), the proposed system relies on intelligent algorithms to realize automatic, comprehensive, and quick identification, review, tagging, storage and query of videos and images in media resources. The research findings can effectively improve the quality of college teaching and enhance the value of media resources.
\end{abstract}

Keywords-Cloud service, artificial intelligence (AI), media resource management (MRM), colleges

\section{Introduction}

The development of the Internet and digital media, coupled with the proliferation of the 5th-generation $(5 \mathrm{G})$ mobile network, has given rise to a novel education model called the Internet Plus education. Under this model, an abundance of quality resources of higher education is created through all kinds of online open courses, such as the massive online open courses (MOOCs) and the micro-lectures.

Nowadays, colleges carry out various academic activities in smart classrooms (e.g. interactive teaching, webcast teaching, and academic lectures), creating a huge amount of video and image files about teacher-student interactions and other aspects of such activities. These files provide the colleges with valuable information in multiple dimensions, namely, teaching, scientific research, management, and student training.

The values of these video and image resources could be fully tapped by the media resource management (MRM) system. On the one hand, the MRM system can efficiently manage these resources through digital storage, management and application. On the other hand, the MRM system can integrate multiple links like teaching, courseware, production, and broadcast, and release and exchange programs 
in different forms for different organizations and departments, thereby enhancing the value of media resources [1-3].

Many scholars have attempted to develop and construct MRM systems. For example, Wang Jundai designed a channel management system for distributed streaming media, which combines local injection of resources and distributed management of channel information to facilitate the storage of media data. However, the channel management system lacks key functions like media retrieval. Based on the cloud computing center in his college, Zhang Yongbo created a small audio-visual media asset management system, which can be constructed and maintained quickly and easily. But the incoming media resources must be checked manually, pushing up the workload of the editing staff. With the aid of cloud storage technology, Zhou Xiaomei developed a campus media asset management system capable of storing, cataloging and distributing media files. Nevertheless, the system does not support functions like intelligent identification and fast retrieval of media.

Through the above analysis, this paper designs an MRM system based on cloud service. Through cloud service and artificial intelligence (AI), the designed system relies on intelligent algorithms to realize automatic, comprehensive, and quick review, tagging, storage and query of videos and images in media resources, and realizes onestop intelligent management and application of massive media resources [4].

\section{Architecture design of the cloud service-based MRM system}

\subsection{Technical architecture of the system}

Intelligent MRM system technologies: The intelligent MRM system is used to manage massive unstructured data such as audio, video, and graphic image files, realizing functions such as digitized storage, cataloging management, retrieval and query, data publishing, video encoding, input and output, and workflow management, etc. The system intelligently recognizes the content of the video and image documents, using intelligent algorithms, it can realize legality review, automatic analysis, automatic labeling, and automatic classification and retrieval of multimedia content [5]. For the duplicate content in media resources, it automatically identifies and labels them to avoid the occurrence of a large number of repeated interfaces [6]. The proposed system can automatically identify the quality problems of audio and video files in the transcoding process, including black screen, figured screen, green screen, mosaic screen, and blurred screen, etc. The entire system takes a server cluster as the storage and processing center; all kinds of media data are uploaded to the server cluster; at the same time, the cluster also provides various functions such as media resource retrieval, browsing and cataloging management, etc. The proposed intelligent MRM system is shown in Figure 1. 


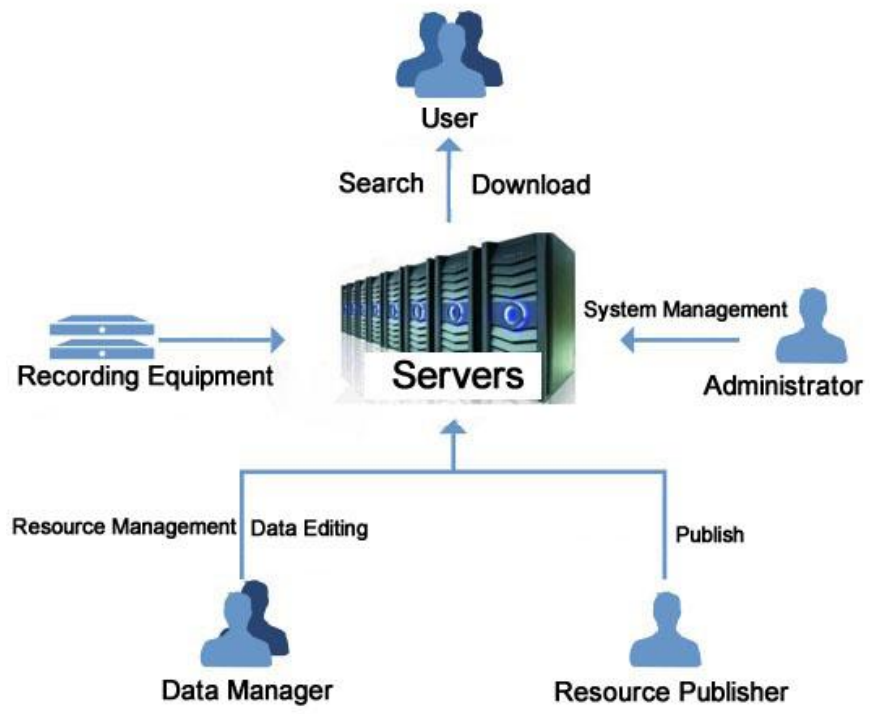

Fig. 1. Intelligent MRM system

Cloud service technology: The traditional system development method often costs a lot of manpower, financial resources and time. According to the requirements of the project, users determine the parameters of hardware equipment, make subsequent purchases, then configure and run the hardware equipment; and this is just the first step of the system operation, regular hardware and software upgrade comes the second, followed by other maintenance tasks such as ensuring the servers are fully compatible with the operating system. All these works have increased the burdens of system developers, making them unable to devote all of their efforts to system software development [7].

Cloud servers can provide IaaS (infrastructure-as-a-service) with excellent performance in stability, reliability, and elastic expansion, freeing users from earlystage preparations such as computer hardware purchase, and realizing instant use and auto scaling of computing resources. Cloud service has the merits of fast delivery and easy deployment, which shortens the launch cycle, transparentizes the operating costs, and supports the expansion and release of resources at any time according to business fluctuations. By accessing other related cloud services through the intranet, various industry solutions could be formed, and the cost of public network data traffic could be reduced. Meanwhile, multiple security solutions such as virtual firewall, access control, intranet isolation, anti-virus, anti-attack and traffic monitoring can be adopted cooperatively.

In system design process, the cloud service platform was taken as the basic underlying architecture, the server clusters in the traditional media resource management system were migrated to the cloud. Developers used the cloud service platform to develop the system, and they only needed to concentrate on data algorithm 
design, computing framework development and AI algorithm training; other tasks such as hardware environment construction, and data transfer and storage were completed by the cloud service platform automatically, which had greatly improved the work efficiency.

\subsection{System architecture design}

The MRM system is a complete set of media digitization solutions which realize unified content management and application through establishing open service platforms. In terms of construction mode, the system adopted the cloud service mode which not only retains the requirements of production in specialization, refinement and security, but also makes good use of the rich content sources on the internet; moreover, the system applied big data technology to realize data analysis and data mining, and used various visualization methods to interpret and store the data, providing convenient services for each school department to utilize the media resources [8].

The proposed system is divided into three layers, namely the basic resource pool layer, the MRM service layer and the business application service layer. The entire system is built by modularized component blocks with good scalability and flexibility. The system architecture is shown in Figure 2.

The first layer, namely the basic resource pool layer, is responsible for providing services such as object storage and file storage, accessing structured data such as stored documents and unstructured data such as images and videos through security mechanisms, and extracting valuable information.

The second layer, namely the intelligent MRM service layer includes three core modules: (1) data processing engine module; (2) metadata management module; and (3) scenario-based packaging module.

1. Data processing engine module: By constructing a computing framework that supports batch asynchronous processing and quasi-real-time synchronous processing, this module can realize rapid and intelligent content review and automatic data processing, and achieve format conversion of multiple media files according to data processing algorithms. It identifies videos and images using AI technologies, then after identifying item tags, detecting human face features, and judging the attributes of items or persons, it achieves automatic machine labeling.

2. Metadata management module: Based on the processing content provided by the data processing engine module, this module uses deep learning algorithms to understand and sort out the image or video scenes, provides external metadata access interface of the scenes, and automatically performs clustering indexing and tag grouping indexing. By constructing metadata sets and calling interface analysis files of tag groups, the obtained metadata are added to the metadata sets, and similar items, environments or persons in the set could be obtained. Through such indexing, rapid tag search such as files could be achieved.

3. Scenario-based packaging module: Through the supports of cloud service to the scenes, the functions of the data processing engine module and the metadata man- 
agement module could be packaged, and services are provided externally in the form of resource packages. The module integrates AI functions such as content recognition, tag detection, and face detection to achieve fast media file retrieval. When school departments need to use the media resources, they can access various resource packages to quickly locate and retrieve the media resources they need.

The third layer, namely the business application service layer, provides end users with web applications and mobile terminal applications.

In the intelligent MRM system, users conduct data communication and transmission with the system through mobile networks, wireless local area networks (WLAN), or wired networks, the technical architecture of the system is shown in Figure 3. Media resource providers can use PCs, mobile terminals, cameras, disks, video tapes and other devices to upload media resources such as videos and images to the intelligent MRM service platform. Then with the help of the powerful computing power of the platform and the AI algorithms, data processing works of the input files such as intelligent review, format conversion, video recognition, face recognition, and label production could be completed within a short time. The distributed service framework of the platform can give full play to the advantages of cloud computing and assign multiple tasks to cloud servers for processing in a balanced manner. Administrator users with resource managing and media editing permissions can access the intelligent MRM service platform through PC and mobile terminals to call all data, and perform data managing, real-time media resource importing, editing and other operations. The data processed by the MRM service platform is then pushed to the basic resource pool through the internal high-speed network of the cloud platform, and operations such as data storage, data label indexing fabrication, and database update are performed according to the relevant data tags. The cloud storage service supports two modes: stream writing and file writing; it could guarantee the reliability of data writing, and seamlessly integrate with the content distribution networks. The virtual distributed network based on the Carrying Network can intelligently cache the content of the source sites, including various dynamic and static resources, to the node servers in various places. This not only facilitates users to obtain content from nearby sites, increases the speed of resource access, but also shares the pressure on the source sites. Finally, the intelligently processed media data can be easily called by terminal applications of multiple users [9]. 


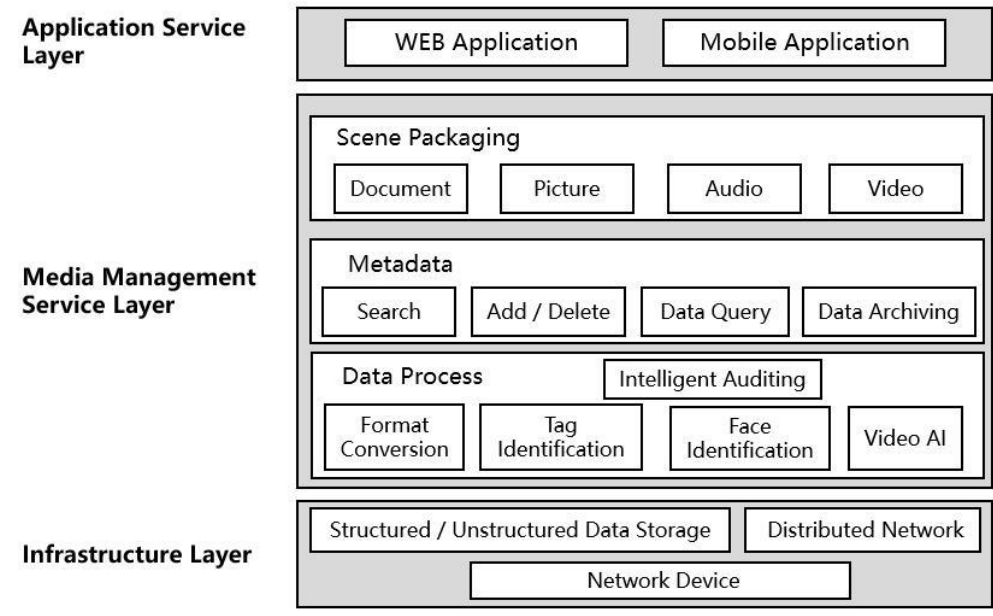

Fig. 2. System architecture

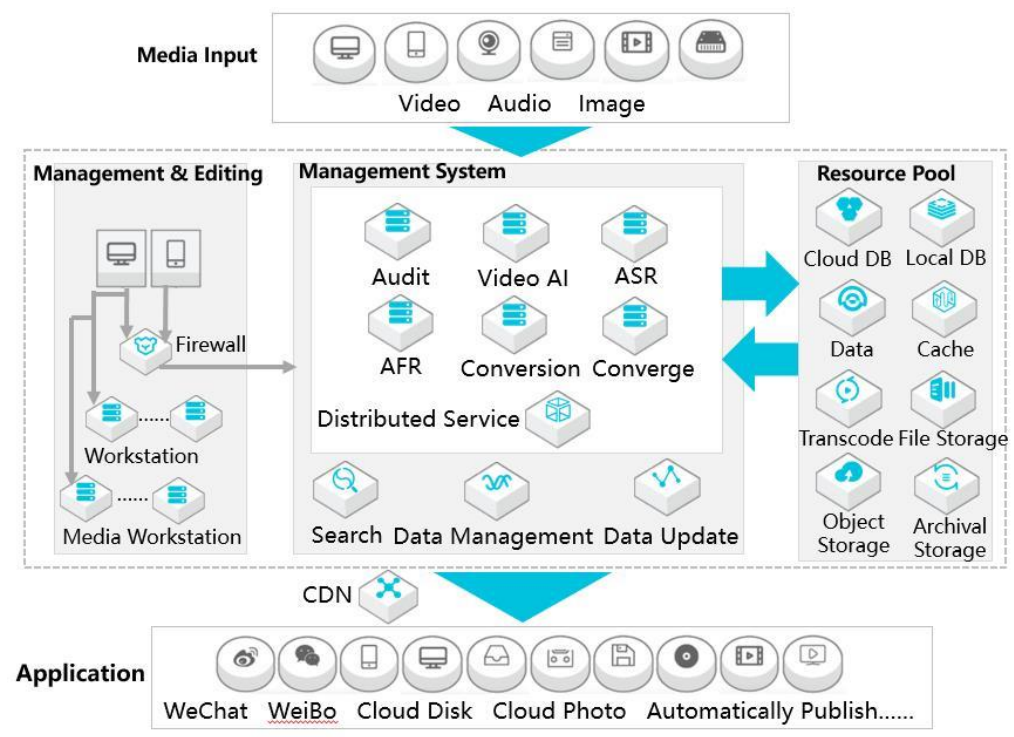

Fig. 3. System technical architecture

\subsection{System operation process design}

The main functions of the intelligent MRM system are to conduct intelligent review, automatic processing and analysis of various media resources, and make good resource indexing for various subsequent applications, therefore, the processing of media resources is the key of design objects, and the work flow of the MRM system is shown in Figure 4. When media resource providers input multiple media resource files, the system first analyzes the category of the input files; if the file format is not 
consistent with the processing file categories, the system will prompt that the input file format is incorrect, the processing cannot be continued, and files that can meet the requirements should be re-entered; if the file has passed the category analysis, it will be pushed to the system for intelligent review, and the images and videos in the sources will be checked for whether there are illegal content in them. If the file contains illegal content, it cannot pass the review; the system will prompt that the file violates the regulations, and give the specific violation content, then the system will require to re-enter the file, if violation prompt appears again, the file will be pushed to the system reviewer administrator for manual review; if the file does contain illegal content, it will be subject to data processing and identification; if the data processing fails, the system will prompt error information and the secondary processing will be performed; if the data processing and identification are successful, then the format of the file will be converted, the metadata information in the file will be detected using the front-end preview engine technology, the content of the file will be marked, and the index will be added to the MRM system to facilitate subsequent business services and application calls.

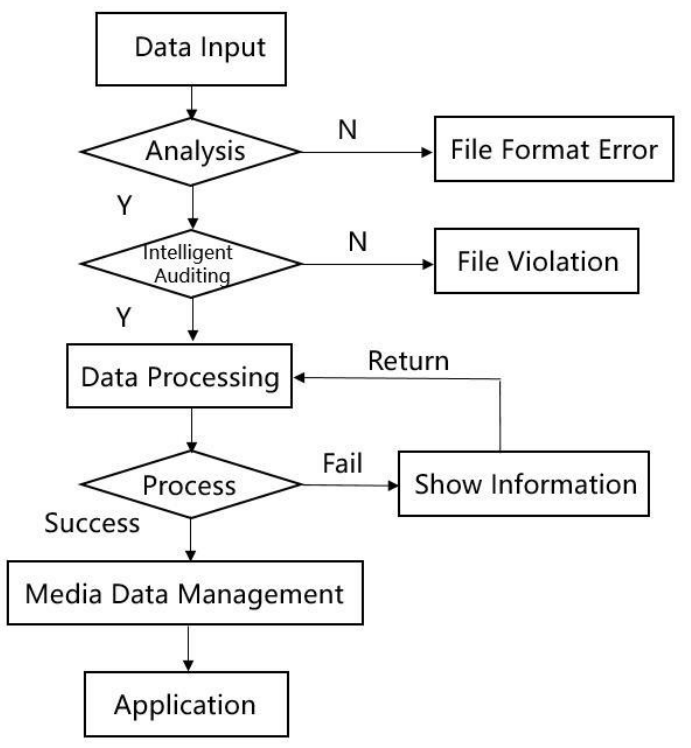

Fig. 4. Resource processing flow of the intelligent MRM system

\subsection{Data tag design of the intelligent MRM system}

The intelligent MRM system helps users to manage, search, and edit media resources. Since media files generally contain various information, metadata has been adopted for management. The system performs various AI recognition and detection operations on the media files, such as face recognition, item recognition, tag detection, etc., and standardizes the analysis result information and saves them in the metadata. In subsequent use, the information can be used as conditions to perform 
quick search and other operations on the media files in the set. The created metadata set is shown in Table 1.

Table 1. Metadata set

\begin{tabular}{|l|l|c|l|}
\hline \multicolumn{1}{|c|}{ Parameter Name } & \multicolumn{1}{c|}{ Data Type } & Key & \multicolumn{1}{c|}{ Description } \\
\hline Project & string & Y & Project Name \\
\hline SetId & string & & Metadata Set ID \\
\hline SetName & string & & Metadata Set Name \\
\hline RequestId & string & & User Identification Code \\
\hline CreateTime & string & & Creation Time \\
\hline
\end{tabular}

\section{Implementation of the MRM System Based on Cloud Services}

\subsection{Operation of the cloud service-based MRM system}

The intelligent MRM system analyzes and converts the input media resources; it constructs a metadata set and uses AI algorithms to analyze the video and image files, then the uploaded data is added to the metadata set, so that a summary of similar categories in the set can be obtained. First, the system remotely connects to the cloud service address; then, a metadata set is created in the management system, and the ID of the dataset is returned, meanwhile the corresponding project progress is created as well. After that, the AI multimedia interface is called to index the multimedia data to be analyzed to the metadata set, thereby completing the final indexing work.

The followings are the key code parameters, during application, other public parameters are also required to complete the call.

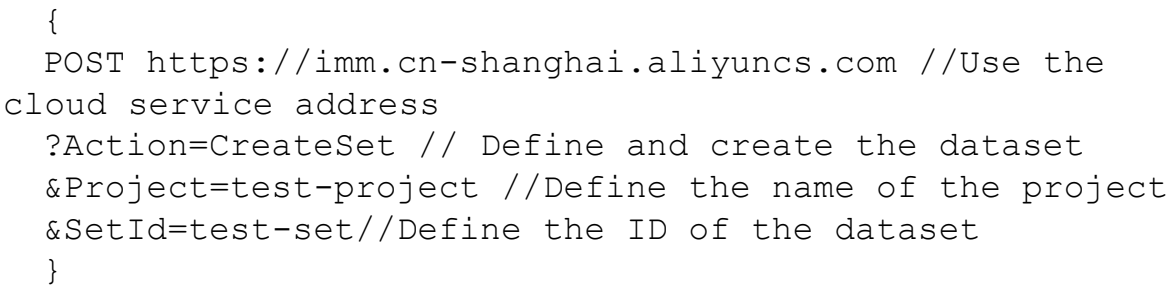

\subsection{Implementation of various functions of the cloud service-based MRM system}

The intelligent MRM system can transfer, manage and edit media files through PC and mobile terminals [10]. Its main functions include:

1. Multi-terminal input and editing: the system was developed based on multiple platform terminals, users can use FTP to upload media resources such as videos and images using PC and mobile terminals through wireless network, 4G or 5G mobile networks; the system supports PC and mobile terminals to conduct online editing, preview, and secondary release of media resources in the system library. 
2. AI review: based on massive tagged data and deep learning algorithms, the system can accurately identify the input videos, images and other resources in real time, and quickly and intelligently determine whether there are prohibited content in the resource. After the review is passed, it enters data processing, storage module, business platform and other processing procedures.

3. AI recognition: using AI recognition technology, the system intelligently identifies and automatically labels the figures, objects, videos, and transitions in various media resources. During detection, the system can identify multiple figures or objects, mark them with rectangular boxes, and generate metadata based on their attributes. The AI algorithm classifies similar figures or items in multiple media files, constructs a metadata set, and adds the obtained metadata to the metadata set, so that similar figures or items in the set can be obtained. Meanwhile, the system can also automatically detect item tags and related confidence in media data, and perform searches and statistics.

4. Deep content search: based on the AI recognition results, the system can automatically construct tagged data, intelligently make catalogues, achieve rapid information extraction of media content such as videos and images; through multiple methods such as keywords, face recognition, and image and video extraction, the system can realize quick search of media resource content and second-level querying of massive content library.

5. Media processing: namely the multimedia data processing service. The system makes use of the elasticity of the cloud computing and its highly scalable characteristics to perform transcoding operations quickly and efficiently during the storage process; when reading, the stored video files could be quickly transcoded into formats with multi-rates and high resolution that are suitable for playing on PC and mobile terminals.

6. Media resource storage: the storage system is divided into three types: standard, low-frequency access, and archiving. The standard storage module has highthroughput and low-latency, its service response capabilities can support frequent access to various types of hotspot data. The low-frequency access storage module is used for long-term storage of infrequently accessed data, it supports real-time data access, and suitable for long-term backup of media data. The archiving storage is suitable for archived data that needs to be stored for a long time and is rarely accessed during the storage period.

7. Intelligent media editing: the system uses intelligent algorithms to analyze video content such as teaching, lectures, meetings, etc., conduct smart positioning, output time stamps, filter the time stamps according to the types of the video, and automatically make video clips. During secondary editing, the system can intelligently search for materials in the system resource library that are suitable for the theme according to manually entered keywords, pack the generated video products, shorten the video editing time, and quickly share the edited materials through the application programs to different platforms. 


\subsection{Realization of the AI functions of the cloud service-based MRM system}

The intelligent MRM system uses AI algorithms to analyze multi-modal information such as videos and images, automatically output multi-dimensional content tags of the videos and images, and convert unstructured information into structured information. Using AI-based target detection technology, it can accurately find out the multiple targets and positions in given videos and images, and give the specific category of each target [11].

Convolution Neural Network (CNN) has made rapid progress in the field of image recognition [12-14]. CNN takes the information of the image pixels as input, extracts and abstracts the features through convolution, and directly outputs the results of image recognition. It can preserve the original information of the images to a great extent, enabling the end-to-end learning method to achieve good results. Inception is a special and powerful CNN that can make use of the high computational performance of the dense matrices while maintaining the sparsity of network results, so as to improve the generalization ability of the model.

Visual modeling of the cloud service platform has been adopted for modeling. The platform applies the visualized drag-and-drop layout, and integrates various data sources, components, algorithms, models and evaluation modules to support the entire development process from data preprocessing, model training, to model evaluation. The Inception algorithm was applied to build an image classification model, by dragging corresponding components, the model was constructed rapidly within a short time, and the actual problems under classified image scenes were solved. In later stages, the parameters can be adjusted and optimized for specific results. A diagram of the visual modeling is shown as Figure 5.

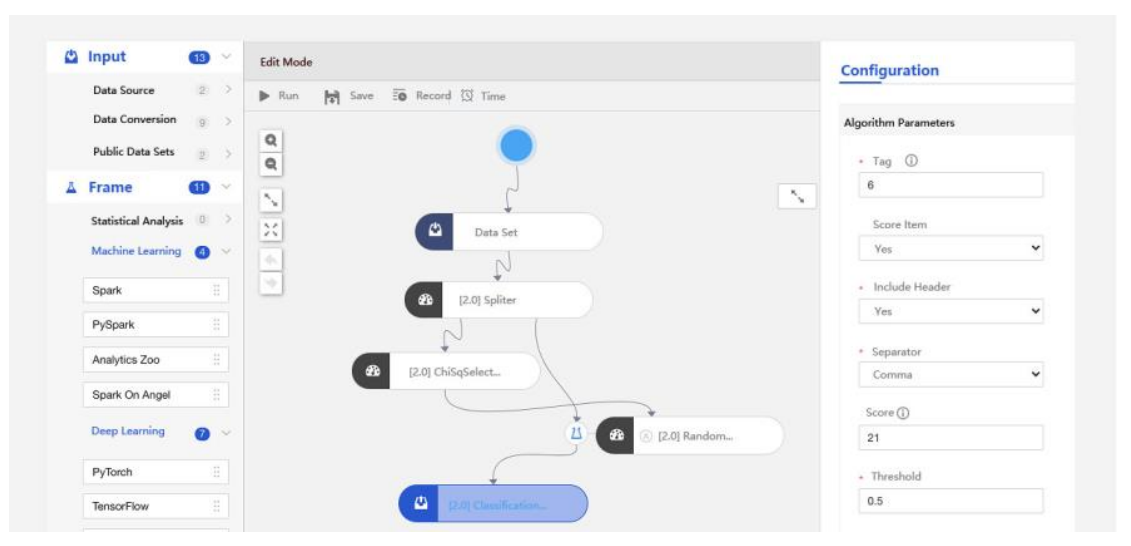

Fig. 5. Visual modeling of the cloud service platform

In AI processing, the most important thing is data algorithm modeling.

Step 1: The cloud service platform creates a new project and task flow.

Step 2: Data access, upload the video and image dataset directly from the local to the cloud service platform or import from the cloud storage space. 
Step 3: Data preprocessing, preprocess the missing, duplicated data, and other problems in the dataset, and convert the strings into numerical forms that can be used in modeling. The data is divided into a training set, a validation set, and a test set. The training set is used to train the model, the validation set is used to adjust the model parameters, and the test set is used to evaluate the overall performance of the model.

Step 4: Model training. The cloud service platform is equipped with a variety of built-in machine learning and deep learning operators, in the visual modeling interface, select suitable operators for modeling, drag the chosen operators into the canvas and connects them according to the node schema.

Step 5: Model evaluation, use the model evaluation operator of the cloud service platform to check the training results of the model.

Step 6: Run the workflow, click the "Run" above the canvas to run the workflow. After the model training effect meets expectations, it can be launched in real use officially.

\section{Test of the Cloud Service-Based MRM System}

The intelligent MRM system conducts data model training on imported images, audios and videos, automatically recognizes, classifies, and detects the graphics images and their contained objects, generates the customized business model, and uses iterations to continuously improve the its precision and recall rate, and accuracy; finally, the system applies the model to actual business, so as to realize automatic recognition, classification and labeling of files of various formats such as images and videos.

\subsection{Model evaluation and test of the intelligent MRM system}

Taking the image recognition data model as an example, first, the data model is selected, and the image groups that have been marked with attributes and classified according to the sport category have been uploaded. After all the data has been uploaded, the chosen data model is subject to AI model training. After the training results are returned, the recognition accuracy, recall rate and other data could be read from the console. If the training result or verification result is unsatisfactory, new images can be added to the training set, re-labeled, and the model training and iteration can be performed again. In model training, the more the categories of images in the training set, the higher the accuracy of image recognition and labeling of the system when it finally launches in real use. The accuracy rate of the training model refers to the percentage of the number of samples with a certain label that have been correctly predicted to the total number of samples that have been predicted to have the label. The recall rate refers to the percentage of the number of samples with a certain label that have been correctly predicted to the total number of samples with the label. Higher accuracy and recall rate indicate that the data model designed by the user meets the business expectations and can be directly released and deployed. After the 
model is released, it can be used to quickly identify and label the sports images. The verification of the model training results is shown in Figure 6.

\subsection{Automatic identification test of media resource content}

Taking ski images as an example, after the user has imported the images into the intelligent MRM system, first, the system conducts format review and content review, after the reviews are passed, the AI content recognition data model is called to automatically identify and label the images, mark the item tags in the images and the confidence values. In this test, after the system had automatically identified the image content, the images were labels with 6 tags of ski, winter sports, ski mountaineering, etc., and the confidence intervals were marked as well. Later, when user searches for keywords such as "ski", "winter sports" and "ski mountaineering" in the media resources, the system can automatically call up the images to match the searches. The automatic identification of media resources is shown in Figure 7.

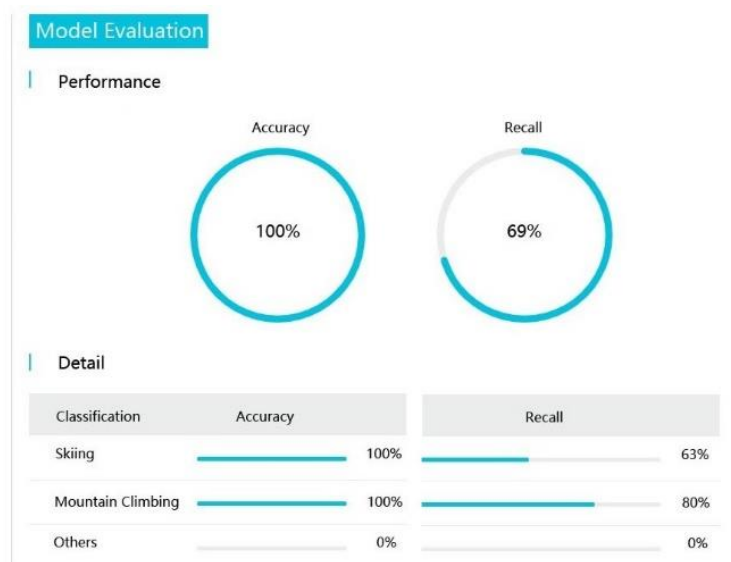

Fig. 6. Model training result verification
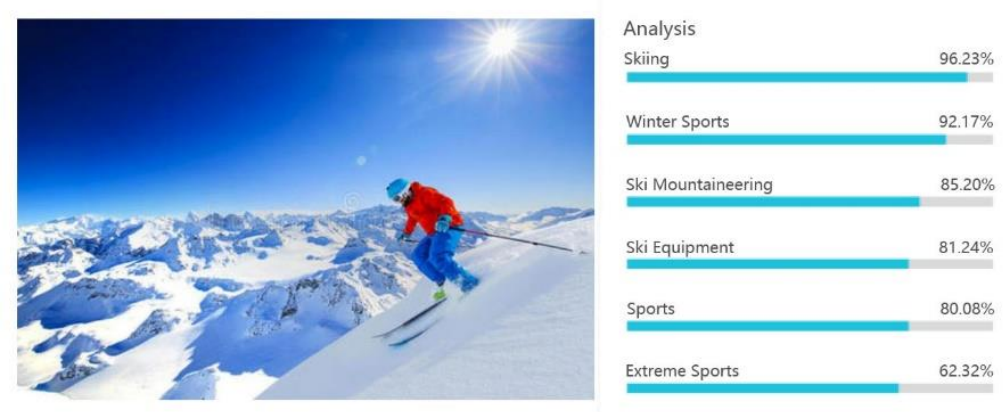

Fig. 7. Automatic identification of media resources 


\section{Conclusion}

This paper proposed an MRM system solution based on cloud services. The system was developed using cloud service platform technologies, and has flexible expansion capacity; cooperated with powerful middleware and big data analysis technologies, relational, non-relational and cached databases are adopted according to different data structures in the actual business. The system replaces the burdensome manual review, automatically analyzes the audio and video content, and meanwhile taps new values of the data to help colleges and universities transform from the traditional media to the cloud service management, thereby realizing the full life cycle management of media resource such as collection, review, storage, editing, production, and distribution.

With the digital and networked development of higher education, the cloud service-based MRM system can effectively collect and manage the ever-increasing resources of teaching, scientific research videos, images, etc., bringing convenience to the management of media resources in colleges and universities, improving work efficiency, providing new services, developing new business areas, better exerting the role of technology platforms, reducing media operating costs of colleges and universities, improving overall competitiveness and information service levels, and ultimately realizing the value of media resources to the greatest extent.

\section{Acknowledgement}

This work was supported in part by Research and Development Planning Project of Shandong Province, China (Year: 2019; No: 2019GGX105019); Education and Teaching Reform Research Project of Shandong University (Year: 2020; No: 2020Y288); Construction and Management Research for Laboratory Major Project of Shandong University (Year: 2020; No: SY20201101).

\section{$7 \quad$ References}

[1] Maya, E., Dominguez-Limaico, H.M., Jaramillo-Vinueza, D., Vasquez-Ayala, C., CastroAndrade, P. (2020). University Applications Based on Cloud Computing: North Technical University Case. Technology, Sustainability and Educational Innovation (TSIE). Advances in Intelligent Systems and Computing, 244-253. https://doi.org/10.1007/978-3-030-37221$\underline{721}$

[2] Rahmelina, L., Firdian, F., Maulana, I.T., Aisyah, H., Na'am, J. (2019). The effectiveness of the flipped classroom model using e-learning media in introduction to information technology course, International Journal of Emerging Technologies in Learning, 14(21), 148162. https://doi.org/10.3991/ijet.v14i21.10426

[3] HimaBindu, G., Anuradha, C., Chandra Murty, P.S.R. (2019). Assessment of combined shape, color and textural features for video duplication. Traitement du Signal, 36(2), 193199. https://doi.org/10.18280/ts.360210 
[4] Chiramdasu, R. (2019). Extended statistical analysis on multimedia concealed data detections. Ingénierie des Systèmes d'Information, 24(2), 161-165. https://doi.org/10.182 $\underline{\text { 80/isi.240205 }}$

[5] Fu, H.H., Xu, J., Zhang, H., Zhang, M., Xu, X.X. (2018). A novel video target tracking method based on lie group manifold. Traitement du Signal, 35(3-4), 331-340. https://doi. org/ 10.3166/TS.35.331-340

[6] Jiang, Y. (2019). Wireless resource management mechanism with green communication for multimedia streaming. Multimedia Tools and Applications, 78(7): 8699-8710. https:// doi.org/10.1007/s11042-018-6149-4

[7] Sarode, N., Bakal, J.W. (2020). A Review on use of Mobile Cloud System in Educational Sector. 2020 6th International Conference on Advanced Computing and Communication Systems (ICACCS), 715-720. https://doi.org/10.1109/icaccs48705.2020.9074167

[8] Kanbul, S., Kallagov, T.E., Rubanik, V.E., Khairullina, E.R., Ribakova, L.A. (2019). Determination of mobile addiction and social media addiction level of parents and their attitudes towards usage of technology. International Journal of Emerging Technologies in Learning, 14(22), 175-191. https://doi.org/10.3991/ijet.v14i22.11770

[9] Ali Ahmed, E., Ali Ahmed, H. (2018). A Proposed Model for Education System Using Cloud Computing, 2018 3rd International Conference on Emerging Trends in Engineering, Sciences and Technology (ICEEST), Karachi, Pakistan, 1-4. https://doi.org/10.1109/iceest. 2018.8643331

[10] Jin, H.X., Fu, Y.Y., Yang, G.L., Zhu, X.N. (2020). An intelligent scheduling algorithm for resource management of cloud platform. Multimedia Tools and Applications, 79(7-8): 5335-5353. https://doi.org/10.1007/s11042-018-6477-4

[11] Liu, C. (2014). A cloud-computing-based resource allocation model for university resource optimization. International Journal of Grid and Distributed Computing, 7(3): 113-122. https://doi.org/10.14257/ijgdc.2014.7.3.11

[12] Ye, Z.X., Chen, Q., Zhang, Y., Zou, J.F., Zheng, Y. (2019). Identification of vortex structures in flow field images based on convolutional neural network and dynamic mode decomposition. Traitement du Signal, 36(6), 501-506. https://doi.org/10.18280/ts.360604

[13] Chirra, V.R.R., Uyyala, S.R., Kolli, V.K.K. (2019). Deep CNN: A machine learning approach for driver drowsiness detection based on eye state. Revue d'Intelligence Artificielle, 33(6), 461-466. https://doi.org/10.18280/ria.330609

[14] Bansal, N., Sharma, A., Singh, R.K. (2019). An evolving hybrid deep learning framework for legal document classification. Ingénierie des Systèmes d'Information, 24(4), 425-431. https://doi.org/10.18280/isi.240410

\section{Authors}

Heng Sun is with the Modern Educational Technology Lab, School of Foreign Languages and Literature, Shandong University, Jinan, Shandong, China. His research directions are computer technology application, network information technology, communication technology and modern education information. (E-mail: sunheng@sdu.edu.cn) 
Wan Ni is the corresponding author of this paper, he works in the School of Journalism and Communication, Shandong University, Jinan, Shandong, China. His research directions are research on image communication and research on network new media. (E-mail: niwan@sdu.edu.cn)

Peng Zhao is with the Modern Educational Technology Lab, School of Foreign Languages and Literature, Shandong University, Jinan, Shandong, China. His research directions are computer technology application and modern education information. (E-mail: pender521@ sdu.edu.cn)

Article submitted 2020-09-04. Resubmitted 2020-10-13. Final acceptance 2020-10-14. Final version published as submitted by the authors 